\title{
Assessment of Physico-Chemical and Microbiological Quality of Drinking Water in Three Upazilas of Noakhali District in Bangladesh
}

\author{
Md. Ariful Islam, Supriya Ghosh, Anwar Ul Alam, Md. Tazul Islam, \\ Md. Ruhul Kabir, Marium Sultana
}

Department of Food Technology and Nutrition Science, Noakhali Science and Technology University, Noakhali, Bangladesh

Email: arifulislam.im@gmail.com

How to cite this paper: Islam, M.A., Ghosh, S., Alam, A.U., Islam, M.T., Kabir, M.R. and Sultana, M. (2021) Assessment of Physico-Chemical and Microbiological Quality of Drinking Water in Three Upazilas of Noakhali District in Bangladesh. Open Access Library Journal, 8: e7941.

https://doi.org/10.4236/oalib.1107941

Received: September 10, 2021

Accepted: October 12, 2021

Published: October 15, 2021

Copyright (c) 2021 by author(s) and Open Access Library Inc.

This work is licensed under the Creative Commons Attribution International License (CC BY 4.0).

http://creativecommons.org/licenses/by/4.0/

\begin{abstract}
This study was driven for analyzing the drinking water quality of three upazillas at Noakhali district in Bangladesh. Nine different areas drinking water samples were collected from three upazilas (sadar-upazila, kabirhat upazila, Chatkhil upazila). Physical \& chemical parameters of the collected samples like $\mathrm{pH}$, electrical conductivity, salt, TDS, chloride-ion, iron content were measured and microbial parameters like Total Viable Bacterial Count (TVBC), Most Probable Number (MPN) of coliforms, E. coli, Pseudomonas, Klebsiella, Enterobacter aerogenes were also detected. All the parameters were compared with WHO drinking water quality standard ranges to understand the condition of drinking water in these areas. This study showed the $\mathrm{pH}$ of Chatkhil upazila was comparatively lower (6.18) than other upazilas. Electrical conductivity was also lower $(252.1 \mu \mathrm{S} / \mathrm{cm})$ in Chatkhil than other upazilas. Salt volume was comparatively higher $(554.33 \mathrm{ppm})$ in sadar upazila sample than in others. TDS of all upazila samples were in an acceptable range. Chloride-ion was very much higher $(7326.33 \mathrm{mg} / \mathrm{L})$ in upazila-sadar. Iron content was mostly $(3 \mathrm{mg} / \mathrm{L})$ in Chatkhil upazila. Total bacterial load was comparatively higher $\left(7.13 \times 10^{6} \mathrm{CFU} / \mathrm{mL}\right)$ in Kabirhat upazila. Most Probable Number (MPN) of Coliforms were comparatively higher (24.67 number of coliforms $/ 100 \mathrm{~mL}$ ) in upazila-sadar than others. This study also showed the water samples that were collected almost all were contaminated by $E$. coli bacteria without supply water in upazila-sadar. As a result, water was not suitable for drinking. Water purification is very urgently needed for saving people from water-borne diseases. So necessary steps should immediately be taken for purifying the sources of drinking water.
\end{abstract}




\section{Subject Areas}

Food Science \& Technology

\section{Keywords}

Physical \& Chemical Parameters, Chloride-Ion, Most Probable Number, Serial Dilution, Coliforms, E. coli

\section{Introduction}

Water plays a vital role in maintaining human life and welfare. Pure drinking water is now recognized as a fundamental right of human beings. Around 780 million people do not have access to clean and safe water and around 2.5 billion people do not have proper sanitation. As a result, around 6 - 8 million people die each year due to water-related diseases and disasters [1]. Therefore, water treatment is a top-priority policy agenda in many parts of the world [2]. In today's world, water use in household supplies is commonly defined as domestic water. This water is processed to be safely consumed as drinking water and for other purposes. Water quality and suitability for using are indicated by its taste, odor, color, and concentration of organic and inorganic materials [3]. Contaminants in the water can affect the water quality and consequently human health.

Drinking water is the major need of human life. Freshwater quality is a vital concern for mankind since it is directly linked with public health. Drinking water quality has always been a major issue in many countries, especially in developing countries like Bangladesh [4]. Although safe drinking water is a basic demand for people all over the world, a huge proportion of people in the world are deprived of pure drinking water including Bangladesh [5]. Groundwater is decreasing day by day prominently in Asia, South America, North America and ecosystems are threatened [6].

Bangladesh is a low-lying country and is a delta of great three big rivers-the Ganges, the Brahmaputra and the Meghna. In Bangladesh, there are many sources of drinking water such as surface water, groundwater and rainwater. Surface and groundwater are considered the main sources of drinking water. There is no safe drinking water supply system in rural areas, except in some large cities (such as "WASA" in Dhaka city). In Bangladesh, most people consider that groundwater or water from tube wells is free from contamination. For this reason, Hand pumped tube-well water is used as the primary source of safe drinking water in Bangladesh. About 90\% of the households use this Hand pumped tube-well technology in Bangladesh [7]. In rural area, most people are depended on untreated groundwater and tube wells. It is an estimation that about $11 \%$ of all deaths in the rural area of Bangladesh are caused by diarrheal disease [8]. Recent studies suggest that significant levels of diarrheal disease are caused in part by drinking untreated groundwater [9] [10]. Natural water re- 
sources such as groundwater in coastal areas of Bangladesh are contaminated by salinity and other heavy metal ions because of saltwater intrusion, storm surges and withdrawal of freshwater for using various purposes [11]. In the Noakhali region, contaminated groundwater is used by people for their drinking purposes and is affected by hypertension, heart failure, kidney failure, skin diseases, carcinogenic diseases, diarrhea and other water-borne diseases. For this reason, a detailed study of the drinking water quality of the Noakhali region is very much important [12]. Although several reports on the assessment of drinking water quality based on physiochemical and Microbiological parameters in the Noakhali region have been published by several researchers separately [5] [12] [13]. Very little information is available about the overall status of water quality in this area. That's why an investigation was initiated, whose primary objective was to examine the present overall status of drinking water quality of groundwater sources in Noakhali region.

\section{Materials and Methodology}

\subsection{Study Areas}

In this study, three upazillas of Noakhali district in Bangladesh drinking water were analyzed. Three upazilas were upazila-sadar, Kabirhat upazila, Chatkhil upazila (Figure 1). Coordinates of Noakhali upazila-sadar is from $22^{\circ} 50^{\prime} \mathrm{N}$ to $22^{\circ} 833^{\prime} \mathrm{N}$ and from $91^{\circ} 6^{\prime} \mathrm{E}$ to $91^{\circ} 100^{\prime} \mathrm{E}$. Coordinates of Kabirhat upazila is from $22^{\circ} 50^{\prime} \mathrm{N}$ to $22^{\circ} 833^{\prime} \mathrm{N}$ and from $91^{\circ} 12^{\prime} \mathrm{E}$ to $91^{\circ} 200^{\prime} \mathrm{E}$. And Chatkhil upazila's coordinate is from $23^{\circ} 3^{\prime} \mathrm{N}$ to $23^{\circ} 5^{\prime} \mathrm{N}$ and from $90^{\circ} 57.5^{\prime} \mathrm{E}$ to $90^{\circ} 95^{\prime} \mathrm{E}$.

\subsection{Sampling}

Total samples were nine in numbers. In upazila-sadar maximum people take supply water as drinking water. Some take motor water and some others take tube-well water for drinking. Drinking water samples were collected from three different areas of Noakhali upazila-sadar. The first sample was collected from maijdee and it was supply water (1000 feet deep and treated). The second sample was collected from Dattabari and it was motor water (500 feet deep). The third sample was collected from Sonapur and it was tube-well water (450 feet deep).

Most of the people at Kabirhat upazila take tube-well water for drinking. From kabirhat upazila three different areas drinking water samples were collected. The first sample was collected from Kabirhat-hospital road and it was tube-well water (60 feet deep). The second sample was collected from Kabirhat-kalamunshi road and it was tube-well water (70 feet deep). The third sample was collected from Kabirhat-college road and it was tube-well water (150 feet deep).

All most the people at Chatkhil upazila take tube-well water for drinking. From Chatkhil upazila three different areas drinking water samples were collected. The first sample was collected from Chatkhil thana road and it was tube-well water (35 feet deep). The second sample was collected from Abu tara nagar road and it was tube-well water (256 feet deep). The third sample was 


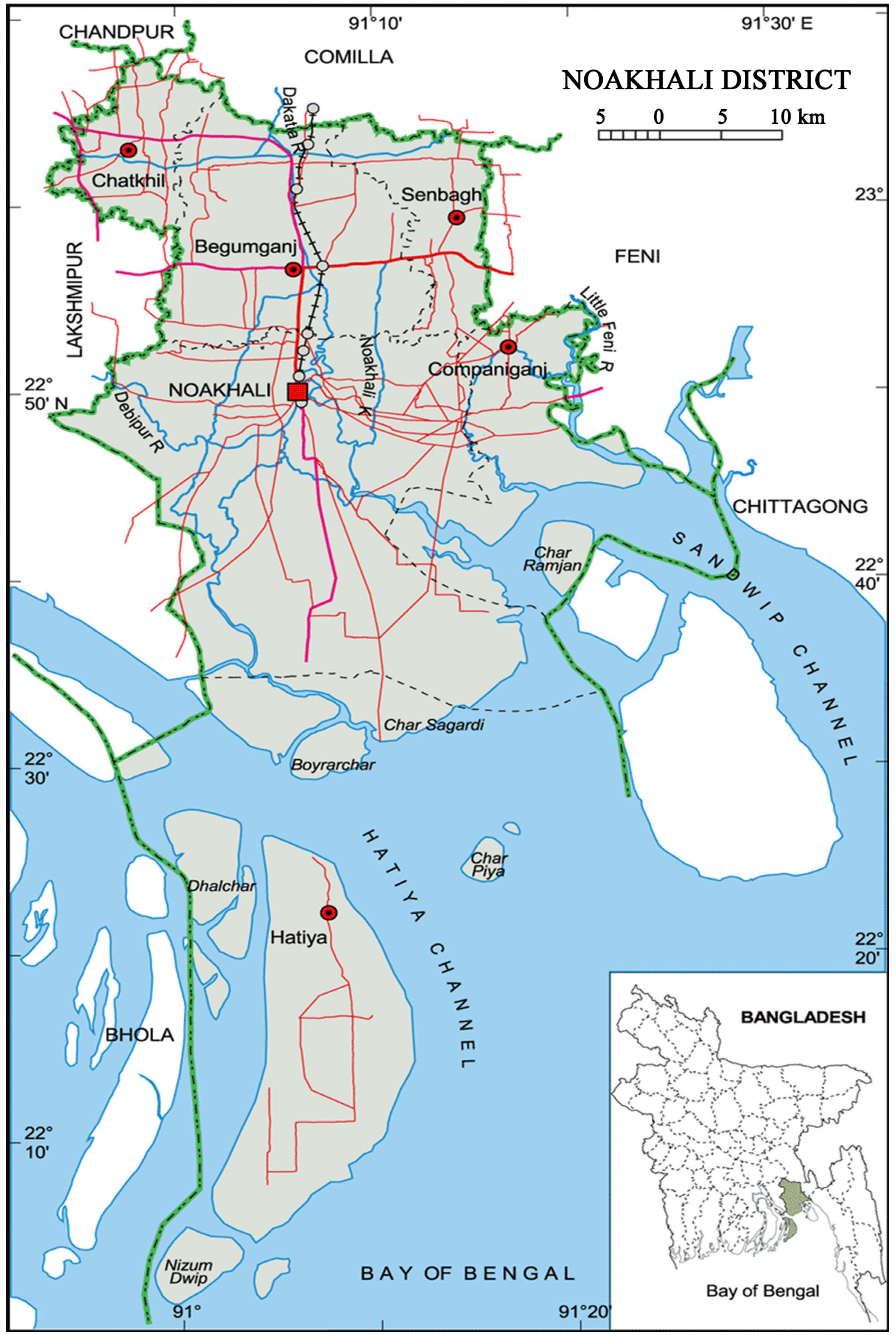

Figure 1. Noakhali district map. 
collected from Bodolkot road and it was tube-well water (800 feet deep).

\subsection{Study Duration}

This study duration was from July to September 2018.

\subsection{Analysis of Physical Parameters}

The $\mathrm{pH}$ values, temperature, total dissolved solid, electrical conductivity (EC), salinity of the samples was measured by using pre-calibrated Multipara-meter, Model PL-700ALS.

\subsection{Determination of Chemical Parameters}

Chloride ion determined in a drinking water sample by "Muhr's Method". Iron content estimated by the packet of reagent in drinking water Sample.

\subsection{Microbiological Test}

Total viable bacterial count by serial dilution process. Total coliform count and E. coli detection by MPN (Most Probable Number) method.

\section{Results}

Physical Test Results: Tables 1-4;

Chemical Test Results: Table 5 and Table 6;

Microbiological Test Results: Tables 7-9.

\section{Discussion}

Drinking water quality at Noakhali district in Bangladesh is not satisfactory. Contamination, high amount of dissolved solids, higher amount of iron content were the regular scenarios. Though supply water was quite satisfactory in upazillas-sadar,

Table 1. Findings of $\mathrm{pH}$ from different drinking water samples of three upazilas.

\begin{tabular}{llll}
\hline Region & Sample-1 & Samlpe-2 & Sample-3 \\
\hline Sadar & 7.77 (1000 feet deep supply water sample) & 7.44 (500 feet deep motor water sample) & $8.22(450$ feet deep tube well water sample) 7.81 \\
Kabirhat 6.72 (60 feet deep tube well water sample) & 6.87 (70 feet deep tube well water) & 6.34 (150 feet deep tube well water) \\
Chatkhil 6.57 (35 feet deep tube well water sample) & 5.81 (256 feet deep tube well water sample) & $6.16(800$ feet deep tube well water sample) & 6.18
\end{tabular}

Table 2. Findings of electrical conductivity from different drinking water samples of three upazilas.

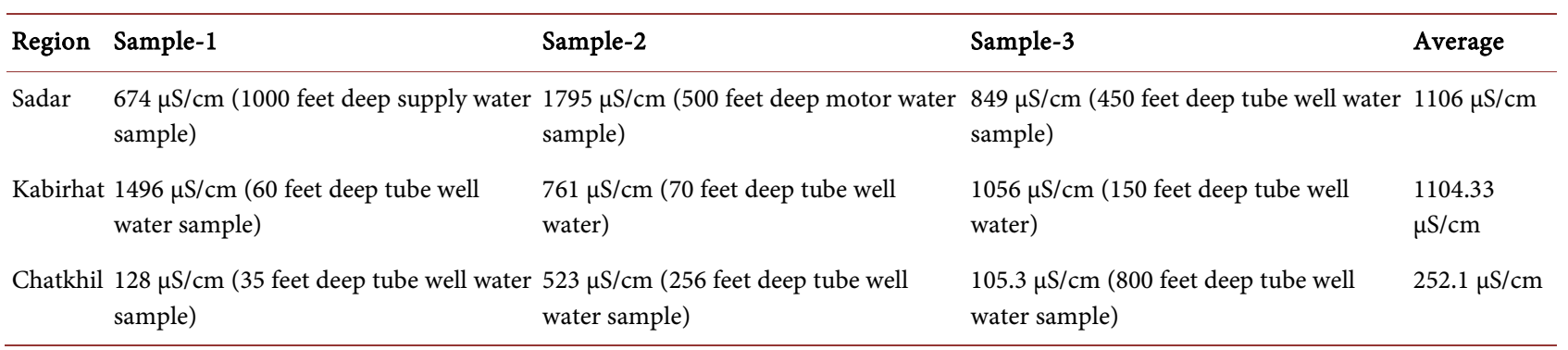


Table 3. Findings of salts from different drinking water samples of three upazilas.

\begin{tabular}{|c|c|c|c|c|}
\hline Region & Sample-1 & Sample-2 & Sample-3 & Average \\
\hline Sadar & $\begin{array}{l}350 \mathrm{ppm} \text { (1000 feet deep supply water } \\
\text { sample) }\end{array}$ & $\begin{array}{l}890 \mathrm{ppm} \text { ( } 500 \text { feet deep motor water } \\
\text { sample) }\end{array}$ & $\begin{array}{l}423 \text { ppm (450 feet deep tube well water } \\
\text { sample) }\end{array}$ & $\begin{array}{l}554.33 \\
\mathrm{ppm}\end{array}$ \\
\hline Kabirhat & $\begin{array}{l}744 \mathrm{ppm} \text { ( } 60 \text { feet deep tube well water } \\
\text { sample) }\end{array}$ & 376 ppm (70 feet deep tube well water) & $527 \mathrm{ppm}$ ( 150 feet deep tube well water) & 549 ppm \\
\hline Chatkhil & $\begin{array}{l}64.1 \mathrm{ppm} \text { ( } 35 \text { feet deep tube well water } \\
\text { sample) }\end{array}$ & $\begin{array}{l}260 \text { ppm ( } 256 \text { feet deep tube well water } \\
\text { sample) }\end{array}$ & $\begin{array}{l}52.3 \mathrm{ppm} \text { (800 feet deep tube well water } \\
\text { sample) }\end{array}$ & $\begin{array}{l}125.47 \\
\mathrm{ppm}\end{array}$ \\
\hline
\end{tabular}

Table 4. Findings of TDS (Total Dissolved Solids) from different drinking water samples of three upazilas.

\begin{tabular}{|c|c|c|c|c|}
\hline Region & Sample-1 & Sample-2 & Sample-3 & Average \\
\hline Sadar & $\begin{array}{l}463 \mathrm{ppm} \text { (1000 feet deep supply water } \\
\text { sample) }\end{array}$ & $\begin{array}{l}1177 \mathrm{ppm} \text { (500 feet deep motor water } \\
\text { sample) }\end{array}$ & $\begin{array}{l}559 \mathrm{ppm} \text { ( } 450 \text { feet deep tube well water } \\
\text { sample) }\end{array}$ & $733 \mathrm{ppm}$ \\
\hline Kabirhat & $\begin{array}{l}983 \mathrm{ppm} \text { ( } 60 \text { feet deep tube well water } \\
\text { sample) }\end{array}$ & 497 ppm (70 feet deep tube well water) & $696 \mathrm{ppm}$ ( 150 feet deep tube well water) & $\begin{array}{l}725.33 \\
\mathrm{ppm}\end{array}$ \\
\hline Chatkhil & $\begin{array}{l}84.5 \mathrm{ppm} \text { ( } 35 \text { feet deep tube well water } \\
\text { sample) }\end{array}$ & $\begin{array}{l}343 \mathrm{ppm} \text { ( } 256 \text { feet deep tube well water } \\
\text { sample) }\end{array}$ & $\begin{array}{l}69.2 \mathrm{ppm} \text { (800 feet deep tube well water } \\
\text { sample) }\end{array}$ & $\begin{array}{l}165.57 \\
\text { ppm }\end{array}$ \\
\hline
\end{tabular}

Table 5. Chloride ion test results from different samples of three upazilas.

\begin{tabular}{|c|c|c|c|c|}
\hline Region & Sample-1 & Samlpe-2 & Sample-3 & Average \\
\hline Sadar & $\begin{array}{l}2836 \mathrm{mg} / \mathrm{L} \text { (1000 feet deep supply water } \\
\text { sample) }\end{array}$ & $\begin{array}{l}14180 \mathrm{mg} / \mathrm{L} \text { ( } 500 \text { feet deep motor water } \\
\text { sample) }\end{array}$ & $\begin{array}{l}4963 \mathrm{mg} / \mathrm{L} \text { ( } 450 \text { feet deep tube well } \\
\text { water sample) }\end{array}$ & $\begin{array}{l}7326.33 \\
\mathrm{mg} / \mathrm{L}\end{array}$ \\
\hline Kabirhat & $\begin{array}{l}1063.5 \mathrm{mg} / \mathrm{L} \text { ( } 60 \text { feet deep tube well } \\
\text { water sample) }\end{array}$ & $2127 \mathrm{mg} / \mathrm{L}$ (70 feet deep tube well water) & $709 \mathrm{mg} / \mathrm{L}$ (150 feet deep tube well water) & $\begin{array}{l}1299.83 \\
\mathrm{mg} / \mathrm{L}\end{array}$ \\
\hline Chatkhil & $\begin{array}{l}354.5 \mathrm{mg} / \mathrm{L} \text { ( } 35 \text { feet deep tube well } \\
\text { water sample) }\end{array}$ & $\begin{array}{l}177.25 \mathrm{mg} / \mathrm{L}(256 \text { feet deep tube well } \\
\text { water sample) }\end{array}$ & $\begin{array}{l}177.25 \mathrm{mg} / \mathrm{L} \text { ( } 800 \text { feet deep tube well } \\
\text { water sample) }\end{array}$ & $\begin{array}{l}236.33 \\
\mathrm{mg} / \mathrm{L}\end{array}$ \\
\hline
\end{tabular}

Table 6. Iron test results from different samples of three upazilas.

\begin{tabular}{|c|c|c|c|c|}
\hline Region & Sample-1 & Sample-2 & Sample-3 & Average \\
\hline Sadar & 0 mg/L (1000 feet deep supply water sample) & $\begin{array}{l}1 \mathrm{mg} / \mathrm{L} \text { ( } 500 \text { feet deep motor water } \\
\text { sample) }\end{array}$ & $\begin{array}{l}2 \mathrm{mg} / \mathrm{L} \text { ( } 450 \text { feet deep tube well water } \\
\text { sample) }\end{array}$ & $1 \mathrm{mg} / \mathrm{L}$ \\
\hline Kabirhat & $2 \mathrm{mg} / \mathrm{L}$ (60 feet deep tube well water sample) & $1 \mathrm{mg} / \mathrm{L}$ (70 feet deep tube well water) & $4 \mathrm{mg} / \mathrm{L}$ (150 feet deep tube well water) & $\begin{array}{l}2.33 \\
\mathrm{mg} / \mathrm{L}\end{array}$ \\
\hline Chatkhil & $2 \mathrm{mg} / \mathrm{L}$ (35 feet deep tube well water sample) & $\begin{array}{l}3 \mathrm{mg} / \mathrm{L} \text { ( } 256 \text { feet deep tube well water } \\
\text { sample) }\end{array}$ & $\begin{array}{l}4 \mathrm{mg} / \mathrm{L} \text { ( } 800 \text { feet deep tube well water } \\
\text { sample) }\end{array}$ & $3 \mathrm{mg} / \mathrm{L}$ \\
\hline
\end{tabular}

Table 7. TVBC (Total Viable Bacteria Count) results from different samples of three upazilas.

\begin{tabular}{|c|c|c|c|c|}
\hline Region & Sample-1 & Sample-2 & Sample-3 & Average \\
\hline Sadar & $\begin{array}{l}1.53 \times 10^{5} \mathrm{CFU} / \mathrm{mL}(1000 \text { feet deep } \\
\text { supply water sample) }\end{array}$ & $\begin{array}{l}1.98 \times 10^{6} \mathrm{CFU} / \mathrm{mL}(500 \text { feet deep } \\
\text { motor water sample) }\end{array}$ & $\begin{array}{l}1.77 \times 10^{6} \mathrm{CFU} / \mathrm{mL} \text { ( } 450 \text { feet deep tube } \\
\text { well water sample) }\end{array}$ & $\begin{array}{l}6.35 \times 10^{6} \\
\mathrm{CFU} / \mathrm{mL}\end{array}$ \\
\hline Kabirhat & $\begin{array}{l}8.5 \times 10^{6} \mathrm{CFU} / \mathrm{mL} \text { ( } 60 \text { feet deep tube } \\
\text { well water sample) }\end{array}$ & $\begin{array}{l}1.13 \times 10^{5} \mathrm{CFU} / \mathrm{mL} \text { ( } 70 \text { feet deep tube } \\
\text { well water) }\end{array}$ & $\begin{array}{l}1.58 \times 10^{6} \mathrm{CFU} / \mathrm{mL} \text { ( } 150 \text { feet deep tube } \\
\text { well water) }\end{array}$ & $\begin{array}{l}7.13 \times 10^{6} \\
\mathrm{CFU} / \mathrm{mL}\end{array}$ \\
\hline Chatkhil & $\begin{array}{l}3.1 \times 10^{6} \mathrm{CFU} / \mathrm{mL} \text { ( } 35 \text { feet deep tube } \\
\text { well water sample) }\end{array}$ & $\begin{array}{l}5.8 \times 10^{6} \mathrm{CFU} / \mathrm{mL} \text { ( } 256 \text { feet deep tube } \\
\text { well water sample) }\end{array}$ & $\begin{array}{l}8.9 \times 10^{7} \mathrm{CFU} / \mathrm{mL} \text { ( } 800 \text { feet deep tube } \\
\text { well water sample) }\end{array}$ & $\begin{array}{l}3.26 \times 10^{6} \\
\mathrm{CFU} / \mathrm{mL}\end{array}$ \\
\hline
\end{tabular}


Table 8. Bacterial Growth presence in E.M.B. (Eosin Methylene Blue) Media from different samples of three upazilas.

\begin{tabular}{|c|c|c|c|}
\hline Region & Sample-1 & Sample-2 & Sample-3 \\
\hline Sadar & $\begin{array}{l}\text { Escherichia coli: Absent } \\
\text { Pseudomonas: Present } \\
\text { Klebsiella: Present } \\
\text { Enterobacter aerogenes: Absent } \\
\text { (1000 feet deep supply water sample) }\end{array}$ & $\begin{array}{l}\text { Escherichia coli: Present } \\
\text { Pseudomonas: Present } \\
\text { Klebsiella: Absent } \\
\text { Enterobacter aerogenes: Absent } \\
\text { (500 feet deep motor water sample) }\end{array}$ & $\begin{array}{l}\text { Escherichia coli: Present } \\
\text { Pseudomonas: Present } \\
\text { Klebsiella: Absent } \\
\text { Enterobacter aerogenes: Absent } \\
\text { (450 feet deep tube well water sample) }\end{array}$ \\
\hline Kabirhat & $\begin{array}{l}\text { Escherichia coli: Present } \\
\text { Pseudomonas: Absent } \\
\text { Klebsiella: Present } \\
\text { Enterobacter aerogenes: Absent } \\
\text { ( } 60 \text { feet deep tube well water sample) }\end{array}$ & $\begin{array}{l}\text { Escherichia coli: Present } \\
\text { Pseudomonas. Absent } \\
\text { Klebsiella: Present } \\
\text { Enterobacter aerogenes: Present } \\
\text { (70 feet deep tube well water) }\end{array}$ & $\begin{array}{l}\text { Escherichia coli: Present } \\
\text { Pseudomonas: Absent } \\
\text { Klebsiella: Present } \\
\text { Enterobacter aerogenes: Present } \\
\text { (150 feet deep tube well water) }\end{array}$ \\
\hline Chatkhil & $\begin{array}{l}\text { Escherichia coli: Absent } \\
\text { Pseudomonas: Present } \\
\text { Klebsiella: Absent } \\
\text { Enterobacter aerogenes: Absent } \\
\text { (35 feet deep tube well water sample) }\end{array}$ & $\begin{array}{l}\text { Escherichia coli: Absent } \\
\text { Pseudomonas: Absent } \\
\text { Klebsiella: Present } \\
\text { Enterobacter aerogenes: Absent } \\
\text { (256 feet deep tube well water sample) }\end{array}$ & $\begin{array}{l}\text { Escherichia coli: Present } \\
\text { Pseudomonas: Absent } \\
\text { Klebsiella: Present } \\
\text { Enterobacter aerogenes: Present } \\
\text { (800 feet deep tube well water sample) }\end{array}$ \\
\hline
\end{tabular}

Table 9. Results of Most Probable Number (MPN) of Coliforms per $100 \mathrm{~mL}$ of different samples of three upazilas.

\begin{tabular}{|c|c|c|c|c|}
\hline Region & Sample-1 & Sample-2 & Sample-3 & Average \\
\hline Sadar & $\begin{array}{l}26 \text { Number of Coliforms } 100 \mathrm{~mL} \text { of } \\
\text { sample water ( } 1000 \text { feet deep supply } \\
\text { water sample) }\end{array}$ & $\begin{array}{l}22 \text { Number of Coliforms } 100 \mathrm{~mL} \text { of } \\
\text { sample water ( } 500 \text { feet deep motor } \\
\text { water sample) }\end{array}$ & $\begin{array}{l}26 \text { Number of Coliforms } 100 \mathrm{~mL} \text { of } \\
\text { sample water ( } 450 \text { feet deep tube } \\
\text { well water sample) }\end{array}$ & $\begin{array}{l}24.67 \text { Number of } \\
\text { Coliforms } / 100 \mathrm{~mL} \text { of } \\
\text { water }\end{array}$ \\
\hline Kabirhat & $\begin{array}{l}17 \text { Number of Coliforms } / 100 \mathrm{~mL} \text { of } \\
\text { sample water ( } 60 \text { feet deep tube } \\
\text { well water sample) }\end{array}$ & $\begin{array}{l}11 \text { Number of Coliforms } / 100 \mathrm{~mL} \text { of } \\
\text { sample water ( } 70 \text { feet deep tube well } \\
\text { water) }\end{array}$ & $\begin{array}{l}14 \text { Number of Coliforms } / 100 \mathrm{~mL} \text { of } \\
\text { sample water ( } 150 \text { feet deep tube } \\
\text { well water) }\end{array}$ & $\begin{array}{l}14 \text { Number of } \\
\text { Coliforms } / 100 \mathrm{~mL} \text { of } \\
\text { water }\end{array}$ \\
\hline Chatkhil & $\begin{array}{l}22 \text { Number of Coliforms/100mL of } \\
\text { sample water ( } 35 \text { feet deep tube } \\
\text { well water sample) }\end{array}$ & $\begin{array}{l}17 \text { Number of Coliforms } 100 \mathrm{~mL} \text { of } \\
\text { sample water ( } 256 \text { feet deep tube } \\
\text { well water sample) }\end{array}$ & $\begin{array}{l}11 \text { Number of Coliforms } 100 \mathrm{~mL} \text { of } \\
\text { sample water ( } 800 \text { feet deep tube } \\
\text { well water sample) }\end{array}$ & $\begin{array}{l}16.67 \text { Number of } \\
\text { Coliforms } / 100 \mathrm{~mL} \text { of } \\
\text { water }\end{array}$ \\
\hline
\end{tabular}

but in other upazillas drinking water were not satisfactory well.

\subsection{PH}

In this study, upazillas-sadar $\mathrm{pH}$ was comparatively higher (7.81) than other upazillas. On the other hand, Chatkhil upazila's drinking water was lower $\mathrm{pH}(6.18)$. Ideal pH level of drinking water is 6.5 - 8.5 (by WHO). Chatkhil upazila's drinking water $\mathrm{pH}$ was under the acceptable range. In that region drinking water was comparatively acidic. The corrosive nature of acidic water causes metal ion such as iron, manganese, copper, lead and zinc to leach into the water causing elevated levels of toxic metals in the water. It may also cause aesthetic problems such as a metallic or sour taste. In previous studies [14] $\mathrm{pH}$ of groundwater was 7.1 to 8.4. Sadar \& Kabirhat upazilas drinking water $\mathrm{pH}$ was similar to the previous studies but Chatkhil upazila's drinking water $\mathrm{pH}$ was comparatively low. The temperature during testing was $26^{\circ} \mathrm{C}-28^{\circ} \mathrm{C}$ those were similar to the previous studies.

\subsection{Electrical Conductivity}

The Electrical Conductivity of drinking water sample at upazillas-sadar was 1106 
$\mu \mathrm{S} / \mathrm{cm}$ which was greater than the acceptable range (Acceptable range 1000 $\mu \mathrm{S} / \mathrm{cm}$ by WHO). Kabirhat upazila's drinking water conductivity was also higher $(1104.33 \mu \mathrm{S} / \mathrm{cm})$ than the acceptable range. Comparatively Chatkhil upazila's drinking water was lower $(252.1 \mu \mathrm{S} / \mathrm{cm})$ than others and was in an acceptable range. Conductivity measures the water's ability to conduct electricity. Common ions in water that conduct electrical current include sodium, chloride, calcium and magnesium. Because dissolved salts and other inorganic chemicals conduct electrical current. Conductivity increases as salinity increases. So, upazila-sadar and Kabirhat drinking water samples had higher salinity. In previous studies [14] Electrical Conductivity of groundwater was $1500 \mu \mathrm{S}$ to $9711 \mu \mathrm{S}$. Sadar \& Kabirhat upazilas drinking water conductivity was a little-bit lower than the previous studies but Chatkhil upazila's drinking water conductivity was comparatively very much low.

\subsection{Salts}

Usually, the standard limit of salinity for drinking water is zero (recommended by WHO, 1996). Upazila-sadar water sample had comparatively higher salt (554.33 ppm) than the other two upazillas water samples. Kabirhat upazila's water sample was also higher salt (549 ppm) than Chatkhil upazila's water sample. Chatkhil upazila's drinking water samples were a comparatively lower amount of salt (125.47 ppm).

\subsection{TDS}

Total dissolved solid below $500 \mathrm{ppm}$ is good for drinking water and the acceptable range is below $1000 \mathrm{ppm}$ by WHO. In this study, upazila-sadar TDS in drinking water were 733 ppm, Kabirhat 725.33 ppm, Chatkhil 165.57 ppm. All three upazillas TDS of drinking water were in acceptable range but not quite good for drinking water. In previous studies [14] TDS of ground water was 128 $\mu \mathrm{S}$ to $1660 \mu \mathrm{S}$. Sadar \& Kabirhat \& Chakhil upazilas drinking water TDS were almost similar to the previous studies.

\subsection{Chloride-Ion}

Chloride ion in upazillas-sadar and Kabirhat upazilas were very high in amount (Sadar $7326.33 \mathrm{mg} / \mathrm{L}$, Kabirhat $1299.83 \mathrm{mg} / \mathrm{L}$ ) which were higher than the acceptable range of $250 \mathrm{mg} / \mathrm{L}$ by WHO. But in Chatkhil chloride ion in drinking water were in the acceptable range $(236.33 \mathrm{mg} / \mathrm{L})$. The high amount of chloride ion in drinking water may wash out beneficiary bacteria from our bodies.

\subsection{Iron}

Concentrations of iron in drinking water are normally less than $0.3 \mathrm{mg} / \mathrm{L}$ by WHO but in this study upazilas-sadar, Kabirhat upazilas, Chatkhil upazila iron content were very much higher than the acceptable range. A high amount of iron may harm our body such as damage healthy skin which can lead to wrin- 
kles. Vegetables and other foods cooked in such water will blacken and absorb a bad taste.

\subsection{Total Viable Bacterial Count (TVBC)}

Total Viable Bacterial Count in Chatkhil upazila drinking water samples bacterial load were comparatively lower $\left(3.26 \times 10^{6} \mathrm{CFU} / \mathrm{mL}\right)$ than others and was almost similar with previous study [14]. In Kabirhat upazila drinking water sample bacterial load was comparatively higher $\left(7.13 \times 10^{6} \mathrm{CFU} / \mathrm{mL}\right)$ than the other two upazilas. According to WHO guideline value, the values of TVBC should remain within $1.0 \times 10^{3}$ but the results of three upazilas showed the values were excessively above the WHO guideline. There is a common belief in Bangladesh that groundwater is relatively free of microorganisms and, therefore, most of the people in rural areas consume tube-well water without any treatment. However, the results of this study show clearly that all water samples that were examined were contained highly. The previous study [14] TVBC of groundwater was 6.73 $\times 10^{3} \mathrm{CFU} / \mathrm{mL}$ in upazila-sadar. All the results of this study were comparatively higher than previous studies.

\subsection{E. coli Detection}

Without supply drinking water in upazila-sadar more or less almost all upazillas had E. coli, Pseudomonas, Klebsiella, Enterobacter aerogenes. These water samples were completely polluted by animal or human waste that may cause serious health hazards like bloody diarrhea, severe anemia and so on. According to WHO guideline value, $E$. coli should be absent in drinking water but the result showed the presence of $E$. coli in every water sample without supply water in upazila-sadar. So, water samples were not suitable for drinking purposes without pretreatment. The previous study [14] E. coli was present in upazila-sadar water sample.

\subsection{MPN (Most Probable Number) Count}

It is a very concern able fact that in upazila-sadar most probable number of $c o$ liforms was $24.67 / 100 \mathrm{~mL}$ which was very much higher than the other two upazilas (Kabirhat $14 / 100 \mathrm{~mL}$, Chatkhil $16.67 / 100 \mathrm{~mL}$ ). Total coliforms include bacteria that are found in water have been influenced by human or animal waste. Fecal coliforms are the group of the total coliforms that are considered to be present specifically in the gut and feces of warm-blooded animals. By these coliforms people may fall into diseases like gastrointestinal illness such as severe diarrhea, nausea and possibly jaundice as well as associated headaches and fatigue.

\section{Conclusion}

Noakhali district is in the coastal area near the Bay of Bengal. As result, the groundwater quality of Noakhali is not good. Higher iron levels in these areas of water are very common. As far as from sea the groundwater iron level, as well as 
microbial load, come low than near areas from sea. The supply water of this district cannot provide to all the people. So, maximum people have to take ground unpurified water for living. For escaping from these problems people should take a suitable water purification method as they can. Otherwise, their health will fall into great difficulties by taking such quality water. Awareness about pure drinking water can make them free from many water-borne diseases.

\section{Conflicts of Interest}

The authors declare no conflicts of interest.

\section{References}

[1] Rahmanian, N., Ali, S.H.B., Homayoonfard, M., Ali, N.J., Rehan, M., Sadef, Y. and Nizami, A.S. (2015) Analysis of Physiochemical Parameters to Evaluate the Drinking Water Quality in the State of Perak, Malaysia. Journal of Chemistry, 2015, Article ID: 716125. https://doi.org/10.1155/2015/716125

[2] Edition, F. (2011) Guidelines for Drinking-Water Quality. WHO Chronicle, 38, 104-108.

[3] Dissmeyer, G.E. (2000) Drinking Water from Forests and Grasslands: A Synthesis of the Scientific Literature. U.S. Department of Agriculture, Forest Service, Southern Research Station, Asheville, 246 p. https://doi.org/10.2737/SRS-GTR-39

[4] Moe, C.L. and Rheingans, R.D. (2006) Global Challenges in Water, Sanitation and Health. Journal of Water and Health, 4, 41-57. https://doi.org/10.2166/wh.2006.0043

[5] Chowdhury, M.M., Kubra, K. and Amin, M.R. (2014) Microbiological Water Pollution in Chittagong Hill Tracts in Bangladesh. International Journal of Medical Science and Public Health, 2, 37-42.

[6] Gleeson, T., Wada, Y., Bierkens, M.F. and van Beek, L.P.H. (2012) Water Balance of Global Aquifers Revealed by Groundwater Footprint. Nature, 488, 197-200. https://doi.org/10.1038/nature11295

[7] Emch, M., Yunus, M., Escamilla, V., Feldacker, C. and Ali, M. (2010) Local Population and Regional Environmental Drivers of Cholera in Bangladesh. Environmental Health, 9, Article No. 2. https://doi.org/10.1186/1476-069X-9-2

[8] Streatfield, K., Persson, L.A., Chowdhury, H.R. and Saha, K.K. (2001) Disease Patterns in Bangladesh: Present and Future Needs. International Centre for Diarrhoeal Disease Research, Bangladesh, Dhaka.

[9] Escamilla, V., Wagner, B., Yunus, M., Streatfield, P.K., van Geen, A. and Emch, M. (2011) Effect of Deep Tube Well Use on Childhood Diarrhoea in Bangladesh. Bulletin of the World Health Organization, 89, 521-527. https://doi.org/10.2471/BLT.10.085530

[10] Escamilla, V., Knappett, P.S., Yunus, M., Streatfield, P.K. and Emch, M. (2013) Influence of Latrine Proximity and Type on Tubewell Water Quality and Diarrheal Disease in Bangladesh. Annals of the Association of American Geographers, 103, 299-308. https://doi.org/10.1080/00045608.2013.756257

[11] Khan, A.E., Ireson, A., Kovats, S., Mojumder, S.K., Khusru, A., Rahman, A. and Vineis, P. (2011) Drinking Water Salinity and Maternal Health in Coastal Bangladesh: Implications of Climate Change. Environmental Health Perspectives, 119, 1328-1332. https://doi.org/10.1289/ehp.1002804

[12] Miah, M.Y., Robel, F.N., Bhowmik, S., Bhattacharjee, S., Paul, S.C., Hossain, M.J. 
and Hossain, M.Z. (2015) Assessment of the Coastal Area Water Quality in Noakhali, Bangladesh. International Journal of Scientific and Engineering Research, 6, 1116-1123.

[13] Mahmud, M.T., Mukharjee, S.K., Khalil, M.I., Rahman, M.A. and Hossen, F. (2016) Physicochemical and Microbiological Analysis of Tube-Well Water from Noakhali District, Bangladesh. World, 3, 50-55.

[14] Prosun, T.A., Rahaman, M.S., Rikta, S.Y. and Rahman, M.A. (2018) Drinking Water Quality Assessment from Groundwater Sources in Noakhali, Bangladesh. International Journal of Development and Sustainability, 7, 1676-1687. 